\title{
Atribuições do farmacêutico no âmbito hospitalar para promoção da segurança do paciente: revisão integrativa da literatura
}

\author{
Attributions of the pharmacist in the hospital scope to promote patient safety: integrative literature \\ review
}

Deberes del farmacéutico en el ámbito del hospital para promover la seguridad del paciente: revisión integrativa de la literatura

\author{
Monique Eva Dias Da Silva \\ ORCID: https://orcid.org/0000-0001-5351-3289 \\ Faculdade Integrada Carajás, Brasil \\ E-mail: silvamoniqueeva@gmail.com \\ Annie Elisandra Mesquita de Oliveira \\ ORCID: https://orcid.org/0000-0003-2165-9210 \\ Faculdade Integrada Carajás, Brasil \\ E-mail: anniefarmahol@hotmail.com \\ Yolanda de Jesus Morais \\ ORCID: https://orcid.org/0000-0001-7105-8267 \\ Faculdade Integrada Carajás, Brasil \\ E-mail: farmaceuticayolandamorais5004@gmail.com
}

\begin{abstract}
Resumo
Introdução: O farmacêutico clínico trabalha promovendo a saúde, prevenindo eventos adverso e intervindo nas prescrições para obtenção de resultados clínicos positivos. Continuamente a monitorização farmacoterapêutica, é capaz de reduzir erros relacionados a medicamentos, aumentando com isso a efetividade e minimizando os riscos da farmacoterapia nesse sentido, esse artigo tem como objetivo identificar produções bibliográficas sobre as atribuições do farmacêutico na promoção da segurança do paciente no âmbito. Método: Trata-se de uma revisão integrativa da literatura baseada na pesquisa bibliográfica. As estratégias de busca permitiram a identificação de 571 artigos nas bases de dados selecionadas. Ao longo das análises dos títulos e resumo, aplicando os critérios de inclusão, 561 artigos foram excluídos. Ao total foram selecionados 10 artigos para análise do texto integral que participaram da revisão sobre o assunto. Utilizando critérios de inclusão e exclusão de artigos, após a leitura e aplicação dos critérios selecionados, 10 artigos foram selecionados por atenderem todos os requisitos. Resultados e discussão: Os resultados salientam que o farmacêutico clínico traz benefícios através da identificação de potenciais eventos adversos relacionados à terapia medicamentosa e consequentemente, contribui para a segurança do paciente no desfecho positivo do paciente. Apesar da importância já bem estabelecida do farmacêutico clínico, os autores, relatam que experiências vêm discretamente sendo relatadas e a importância desses serviços para a criança, adolescente e adulto tem levado a resultados interessantes. Considerações finais: Todos os estudos demonstram que a presença do farmacêutico exerce uma interferência benéfica às prescrições médicas, melhorando assim, a qualidade dos serviços prestados ao paciente, diminuindo o óbito, o número e o tempo de hospitalizações e reduzindo os custos hospitalares.

Palavras-chave: Atribuições do farmacêutico; Ambiente hospitalar; Segurança do paciente.
\end{abstract}

\begin{abstract}
Introduction: The clinical pharmacist works to promote health, preventing adverse events and intervening in prescriptions to obtain positive clinical results. Continuously pharmacotherapeutic monitoring is able to reduce errors related to medications, thereby increasing the effectiveness and minimizing the risks of pharmacotherapy in this sense, this article aims to identify bibliographic productions on the attributions of the pharmacist in promoting patient safety in the context. Method: This is an integrative literature review based on bibliographic research. The search strategies allowed the identification of 571 articles in the selected databases. Throughout the analysis of titles and abstract, applying the inclusion criteria, 561 articles were excluded. In total, 10 articles were selected for analysis of the full text that participated in the review on the subject. Using criteria for inclusion and exclusion of articles, after reading and applying the selected criteria, 10 articles were selected for meeting all requirements. Results and discussion: The results highlight that the clinical pharmacist brings benefits through the identification of potential adverse events related to drug therapy and, consequently, contributes to patient safety in the positive outcome of the patient. Despite the well-established importance of the clinical pharmacist, the authors report that experiences have been discreetly
\end{abstract}


reported and the importance of these services for children, adolescents and adults has led to interesting results. Final considerations: All studies demonstrate that the presence of the pharmacist exerts a beneficial interference to medical prescriptions, thus improving the quality of services provided to the patient, reducing deaths, the number and time of hospitalizations and reducing hospital costs.

Keywords: Pharmacist's assignments; Hospital environment; Patient safety.

\section{Resumen}

Introducción: El farmacéutico clínico trabaja para promover la salud, previniendo eventos adversos e interviniendo en las prescripciones para obtener resultados clínicos positivos. El monitoreo farmacoterapéutico continuo es capaz de reducir los errores relacionados con los medicamentos, aumentando así la efectividad y minimizando los riesgos de la farmacoterapia en este sentido, este artículo tiene como objetivo identificar producciones bibliográficas sobre las atribuciones del farmacéutico en promover la seguridad del paciente en el contexto. Método: Se trata de una revisión integrativa de la literatura basada en la investigación bibliográfica. Las estrategias de búsqueda permitieron identificar 571 artículos en las bases de datos seleccionadas. A lo largo del análisis de títulos y resúmenes, aplicando los criterios de inclusión, se excluyeron 561 artículos. En total, se seleccionaron 10 artículos para el análisis del texto completo que participaron en la revisión sobre el tema. Utilizando criterios de inclusión y exclusión de artículos, después de leer y aplicar los criterios seleccionados, se seleccionaron 10 artículos que cumplían con todos los requisitos. Resultados y discusión: Los resultados destacan que el farmacéutico clínico aporta beneficios a través de la identificación de posibles eventos adversos relacionados con la farmacoterapia y, en consecuencia, contribuye a la seguridad del paciente en el resultado positivo del paciente. A pesar de la importancia bien establecida del farmacéutico clínico, los autores informan que las experiencias se han relatado discretamente y la importancia de estos servicios para niños, adolescentes y adultos ha dado lugar a resultados interesantes. Consideraciones finales: Todos los estudios demuestran que la presencia del farmacéutico ejerce una interferencia beneficiosa en las prescripciones médicas, mejorando así la calidad de los servicios prestados al paciente, reduciendo las muertes, el número y tiempo de hospitalizaciones y reduciendo los costos hospitalarios.

Palabras clave: Atribuciones del farmacéutico; Entorno hospitalario; Seguridad del paciente.

\section{Introdução}

A intervenção farmacêutica, com a contínua monitorização farmacoterapêutica, é capaz de reduzir erros relacionados a medicamentos, aumentando com isso a efetividade e minimizando os riscos da farmacoterapia. Nascimento e Draganov (2015) afirmam que desde remotos tempos, a qualidade em segurança do paciente é alvo de significativo debate pelos estudiosos, pois, inobstante o cuidado humano traga inúmeros benefícios, os erros são cada vez mais frequentes no tocante a assistência prestada aos pacientes.

Para Bueno e Fassarella (2012) a segurança do paciente é compreendida como ações cuja finalidade é impedir, precaver e minimizar os desfechos adversos a partir da assistência de saúde. Dessa forma, os danos causados aos pacientes têm sido discutidos cada vez mais no âmbito hospitalar e não hospitalar.

Segundo o Ministério da Saúde (MS) (2014) a qualidade da assistência prestada nos serviços de saúde é cada vez mais uma exigência da sociedade brasileira, reforçada tanto por compromissos internos, quanto por compromissos externos. Nesse contexto, o MS instituiu o Programa Nacional de Segurança do Paciente (PNSP) em 2013, com o objetivo principal de contribuir para a qualificação do cuidado em saúde, em todos os estabelecimentos de saúde de saúde do território nacional, quer públicos, quer privados, de acordo com prioridade dada à segurança do paciente.

De acordo com as diretrizes do Conselho Federal de Farmácia (CFF), a atenção farmacêutica é caracterizada por ações do farmacêutico, nas quais o paciente é o principal beneficiário. Desta forma, o farmacêutico atua mais efetivamente na assistência ao paciente, responsabilizando-se, junto à equipe multiprofissional, pela segurança e pela efetividade da farmacoterapia. Isto se dá por meio da identificação, da resolução e da prevenção de problemas relacionados a medicamentos.

Partindo das explanações anteriores, esta pesquisa levanta o seguinte problema: como as atribuições do farmacêutico inserido no âmbito hospitalar podem contribuir para a segurança do paciente? 
Este estudo busca subsídios dentro do contexto da segurança do paciente, mais especificamente dentro de estabelecimentos de saúde, partindo-se da hipótese de que as ações farmacêuticas contribuem de forma consistente para a segurança do paciente, principalmente na fiscalização, controle e uso racional de medicamentos.

A pesquisa justifica-se pelo aumento indiscriminado de erros relacionados à prescrição, dispensação e administração de medicamentos por parte da equipe multidisciplinar no âmbito hospitalar. É preciso reconhecer que ações simples de serem implantadas, como a intervenção farmacêutica a partir da análise de prescrições, podem identificar problemas relacionados a medicamentos, prevenindo eventos adversos, agregando imensurável valor na segurança do paciente.

O objetivo desse artigo é identificar produções bibliográficas sobre as atribuições do farmacêutico na promoção da segurança do paciente no âmbito hospitalar, publicadas no período de 2008 a 2018, descrevendo o conhecimento produzido acerca desta temática.

\section{Contexto histórico}

A qualidade em segurança do paciente esteve relacionada aos desafios que os profissionais de saúde devem buscar no aprimoramento de uma assistência livre de danos, norteada por eficácia, eficiência e conhecimento técnico-científico associado a modernas tecnologias. Através deste legado é possível notar que mesmo num contexto assistencial elementar, Hipócrates admitiu que os atos assistenciais são passíveis de equívoco e a segurança do paciente já era vista como prioridade (Wachter, 2010).

Hipócrates, pai da medicina e considerado como um pensador à frente da sua época escreveu a célebre frase, in verbis: "Primum non nocere", que significa primeiro não causar dano. Os pesquisadores que o procedem, na busca pela qualidade em segurança do paciente, remontam do século XIX, quando Florence Nightingale, enfermeira inglesa, foi trabalhar na Guerra da Criméia (1853-1856) e, observando as condições precárias em que os soldados se encontravam, priorizou a segurança dos soldados como fator fundamental para uma boa qualidade nos cuidados prestados. Nightingale, que possuía uma mente avançada para sua época, dotava um vasto conhecimento em ciências, matemática, literatura, artes, filosofia, história, política e economia, constatou que existiam falhas nas condutas profissionais as quais eram um sério problema e alerta para a realidade. Ela classificava como primordial a segurança dos doentes devido à consequências observadas (Nascimento; Draganov, 2015).

Ainda de acordo com Nascimento e Draganov (2015) destacou-se como marco de confluência do movimento mundial acerca do tema segurança e divulgação de um estudo nos Estados Unidos da América, nominado: To err is Human: Buidilng a Safer Health Care System (Errar é Humano: Construindo um Sistema de Saúde mais Seguro), em que relatava a alta taxa de mortalidade nos hospitais deste país ocasionada por erros na assistência à saúde.

Para Bueno e Fassarella (2012) na antiguidade não se possuía uma assistência especializada e mesmo assim não era permissivo errar. À medida que ocorreram os avanços no conhecimento e aumentou a probabilidade de sobrevir erros também. Contrariamente a esse contexto, cada vez mais era adotada a conduta punitiva, de modo a identificar e apontar sempre o indivíduo responsável pelo engano.

Segundo relatos de Wachter (2010) Ernest Codman, um cirurgião de Boston, estudou os desenlaces de pacientes, incluindo falhas no tratamento. Após a criação da Joint Commission on Accreditation oj Healthcare Organizations (JCAHO), em 1918 pelo Colégio Americano de Cirurgiões, surge o primeiro trabalho intitulado de Diseases of Medical Progress, onde mostrou a prevalência e evitabilidade de doenças iatrogênicas. Estas são concebidas como o resultado de um procedimento ou ocorrência prejudicial que não foi uma consequência natural da doença do paciente.

No Brasil, pesquisas nesta área surgiram no início da década de 2000, influenciados pelo panorama mundial cuja temática já vinha e continua sendo amplamente discutida, vide as certificações das instituições de saúde com métodos de avaliação de caráter não-obrigatório e renovável sob a forma de acreditação, demostraram que os processos hospitalares não se 
encontravam organizados e adequados para garantir uma assistência segura e associado a essa complexidade, a limitação humana que, inevitavelmente, tem como efeito os erros (Bueno \& Fassarella, 2012).

Ainda para Bueno e Fassarella (2012) a partir da acreditação foi possível detectar e gerenciar os possíveis erros,

falhas no sistema que ocorre constantemente, seja no cuidado direto ou indireto ao paciente, como também na comunicação entre equipes e que gera um cuidado inseguro.

\section{Segurança do paciente e sua importância}

A segurança do paciente tem sido objeto de preocupação da Organização Mundial da Saúde (OMS), desde o ano de 2002. No Brasil, o MS trabalha para levar esse novo paradigma de segurança a todos os serviços hospitalares.

Mudanças sociais e econômicas, além do crescente avanço científico e tecnológico, vêm impulsionando gestores de saúde a se preocuparem cada vez mais com a qualidade dos serviços. Hordineiramente, a qualidade na saúde vai além do alcance do atendimento dos propósitos organizacionais e do alcance da satisfação dos clientes, sendo necessária, inclusive, a redução dos riscos associados à assistência, o que pode ser traduzido como a promoção da segurança do paciente (Brasil, 2014).

Em 2009, um relatório divulgado pelo Instituto de Medicina dos Estados Unidos (Errar é humano: construindo um sistema de saúde seguro) analisou prontuários de 30.121 internações (6,5\% dos quais provocaram disfunções permanente e 13,6\% envolveram a morte do paciente). Com base nestes resultados, estimou-se que os danos haviam contribuído para as ocorrências de 180.000 óbitos por anos naquele país. Após a publicação desse relatório, tornou-se urgente a redução de eventos adversos em todo o mundo. Em se tratando da assistência a saúde nos hospitais brasileiros, acredita-se que os erros e suas consequências são consideravelmente maiores, devido a vários quesitos (Brasil, 2018).

Para o Conselho Nacional de Secretários de Saúde (2018):

Segurança do paciente são todos os estudos e práticas para a diminuição ou eliminação de riscos na assistência em saúde que podem causar danos ao paciente. A segurança do paciente envolve ações promovidas pelas instituições de saúde e ensino para reduzir a um mínimo aceitável, o risco de dano desnecessário associado ao cuidado de saúde. No Brasil, as metas para a segurança do paciente são baseadas nas metas internacionais da Organização Mundial da Saúde (OMS).

Para Bohrer (2016) a segurança do paciente não significa garantia de cuidado integralmente qualificado, contudo, é um dos pilares que fundamenta a qualidade, visto que os riscos associados ao atendimento na saúde são notórios. Por esse motivo, a busca pelo atendimento seguro deve ser um objetivo incessante, além de um compromisso ético, de busca amplamente disseminada nas organizações de saúde.

Sabendo da importância do tema, o Ministério da Saúde editou a Portaria GM/MS 529/2013, que institui o Programa Nacional de Segurança do Paciente. A Agência Nacional de Vigilância Sanitária (ANVISA) institui as ações para segurança do paciente, conforme RDC n 36/2013 com objetivo de prevenir e reduzir a incidência de eventos que gerem danos ao paciente, adotando como escopo de atuação para os eventos associados à assistência à saúde, as seis metas da OMS. Estas metas estão traduzidas nos 6 protocolos de segurança do paciente nas portarias GM/MS 1377/2013 e GM/MS 2.095/2013.

Estudo realizado nos Estados Unidos da América revela que cada paciente internado em hospital norte-americano está sujeito a um erro de medicação por dia, sendo registrados anualmente, nessas instituições, no mínimo 400.000 eventos adversos evitáveis relacionados a medicamentos. Esses eventos adversos podem verificar-se em todas as etapas da cadeia terapêutica e sua ocorrência aumenta consideravelmente os custos do sistema de saúde (Aspden, 2007).

De acordo com Ferracini (2005) a incorporação de princípios para reduzir erros humanos minimizando os lapsos de memória, promovendo acesso a informações sobre os medicamentos e desenvolvendo padrões internos de treinamento reduz a 
probabilidade de falhas e aumenta a chance de intercepta-las antes de resultar em prejuízo ao paciente. Nesse sentindo, devemse incluir estratégias como a padronização de processos, o uso de recursos de tecnologia da informação, educação permanente e, principalmente, o acompanhamento das práticas profissionais em todas as etapas do processo que envolve o medicamento.

\section{Atuação do farmacêutico frente à segurança do paciente}

Em agosto de 2013 foi publicada a resolução 585 do Conselho Federal de Farmácia (CFF) que regulamenta a atribuição do farmacêutico clínico. O farmacêutico também está inserido na Comissão de Controle de Infecção Hospitalar e no Programa de Controle de Infecção Hospitalar. Através da farmácia hospitalar consegue-se monitorar os antimicrobianos e a utilização de saneantes e germicidas nos diversos setores do hospital (Brasil, 2013).

O exercício profissional do farmacêutico busca a concepção clínica de sua atividade, além da integração e colaboração com os membros da equipe de saúde, cuidando diretamente do paciente. A colaboração profissional do farmacêutico na equipe multiprofissional promove relações e interações nas quais os profissionais poderão partilhar conhecimentos, especialização e habilidades entre si, com o objetivo de proporcionar melhor atenção ao paciente em termos terapêuticos, humanísticos e relativos à segurança (Ribeiro et al., 2015).

O conceito de segurança no uso dos medicamentos não é estático. Ele muda conforme avançam os conhecimentos sobre farmacologia, sendo também alterado, ao longo do tempo, pelas situações desastrosas que acontecem com o uso dos fármacos. Na década de sessenta, a tragédia da focomelia, em bebês de mães que fizeram uso da talidomida, acarretou um efeito colateral benéfico, ao tornar mais rígidas as normas para os ensaios clínicos de drogas e aumentar e melhor definir as responsabilidades dos órgãos governamentais e indústrias farmacêuticas sobre os produtos farmacêuticos lançados no mercado. Nessa década, surgem os programas de farmacovigilância, cujo principal objeto de interesse são as reações adversas a medicamentos que acontecem após o lançamento do produto no mercado. O importante para a farmacovigilância é avaliar a segurança no uso de medicamentos comercializados, tomando-se como base a experimentação dos ensaios clínicos, e, diante de suas limitações metodológicas, estabelecer a vigilância das reações adversas a partir do momento em que os medicamentos passam a ser consumidos em larga escala (Alcantara \& Cassiolato, 2010).

Ainda de acordo com Ribeiro et al., (2015) dentre as atividades realizadas pelos farmacêuticos clínicos estão o processo de avaliação de prescrição de medicamentos antes da dispensação e administração, conforme critérios descritos acima, além da prática da conciliação medicamentosa, validação de suspeitas de reação adversa, promoção da educação aos pacientes e familiares/acompanhantes quanto ao uso de medicamentos.

Segundo Carvalho e Vieira (2002), a magnitude dos erros médicos e eventos adversos com drogas nos Estados Unidos que se encontra por volta de um milhão de pacientes/vítimas representa a quarta causa de óbito neste país expondo um problema de saúde pública.

Os medicamentos ocupam um lugar dominante no sistema de saúde e no tratamento de doenças. A alternativa para a busca da cura é, para muitos, a utilização de medicamentos. Aproximadamente $88 \%$ dos pacientes que procuram o serviço profissional do médico recebem hoje prescrições de medicamentos (Cassiani, 2005).

Ainda de acordo com os estudos de Cassiani (2005):

Administrar medicamentos aos pacientes nas instituições de saúde é um processo complexo, com várias etapas, contemplando uma série de decisões e ações inter-relacionadas que envolve profissionais de várias disciplinas bem como o próprio paciente, necessitando destes conhecimentos atualizados sobre os medicamentos e acesso no momento necessário de informações completas e exatas sobre o paciente. Inicia-se com a seleção e prescrição do medicamento e o envia às clínicas, preparo e administração pela enfermagem que registra e monitora as reações deste medicamento. Os profissionais envolvidos são: o médico, farmacêutico, auxiliar do farmacêutico, enfermeiro e o auxiliar ou técnico de enfermagem. 
No âmbito hospitalar a farmácia hospitalar tem por objetivo garantir o uso seguro e racional dos medicamentos que serão prescritos pelo médico. Para garantir a segurança dos pacientes deve-se fazer um planejamento na compra dos medicamentos e materiais hospitalares. $\mathrm{O}$ farmacêutico tem como objetivo garantir o uso seguro e racional de medicamentos, assim como visa na farmácia clínica que busca ter o máximo rendimento terapêutico, e atender a demanda de medicamentos utilizados pelos pacientes hospitalizados. O farmacêutico hospitalar deixou de ter apenas o papel administrativo de organizar medicamentos e recursos financeiros (Santana; Oliveira \& Ribeiro, 2014).

O farmacêutico contribui na elaboração de protocolos clínicos para profilaxia antimicrobiana e para uso terapêutico em infecções bacterianas, avaliando a qualidade de prescrição, e levando sempre em conta os dados farmacoeconômicos disponíveis. A única maneira de conter a resistência das bactérias é através do uso racional de antimicrobianos (Dantas, 2011).

\section{Eventos adversos relacionados à assistência farmacêutica hospitalar}

Segundo Cassiani (2000) erro de medicação pode ser definido como qualquer evento evitável, que de fato ou potencialmente pode ter causado uso inapropriado do medicamento, resultando em algum dano ao paciente enquanto este está sob o controle dos profissionais de saúde, pacientes ou terceiros. Estes eventos podem estar relacionados à prática profissional, produtos, procedimentos e sistemas. É importante salientar a diferença entre o erro de medicação e a reação adversa a medicamento, a segunda refere-se a características intrínsecas do medicamento, sendo inevitável, enquanto a primeira pode ser evitada. Os erros de medicação podem ocorrem em todas ou em qualquer fase do sistema de medicação.

De acordo estudos e dados de Leape et al., (2006), 11\% do total de erros de medicação ocorreram durante a dispensação de medicamentos e 38\%, durante a administração. Enfermeiros e farmacêuticos interceptam $86 \%$ dos erros de medicação relacionados a erros de prescrição, transcrição e dispensação, enquanto apenas $2 \%$ dos erros de administração são interceptados.

Trabalho realizado por Winterstein et al., (2004) refere que 72\% dos erros de medicação são iniciados durante a prescrição, seguidos da administração (15\%), dispensação (7\%) e transcrição (6\%). No Brasil, estudos sobre os erros de dispensação ainda são incipientes.

Em 2000, a publicação do Institute of Medicine (IOM) intitulada To err is human: building a safer health system atraiu a atenção da opinião pública, cientistas e governo norte-americano. Um dado importante do relatório do IOM é o de que erros de utilização de medicamentos são numerosos. Na maioria dos casos, tais erros são passíveis de prevenção, ilustrando a importância da compreensão de que o uso correto de medicação depende de um processo complexo.

No ambiente hospitalar, para que um erro não ocorra, ou caso ocorra, não alcance o paciente, é necessário que haja atuação efetiva de diferentes elementos. A equipe multiprofissional deve atuar de forma integrada nas etapas de seleção, gestão, prescrição, dispensação e administração de medicamentos. O monitoramento da ocorrência de reações adversas, também, é uma tarefa importante e deve contar com a colaboração de pacientes e seus familiares (Castilho, et al., 2008).

Os potenciais erros na dispensação ou na administração de medicamentos detectados no estudo de Castilho et al., (2008), relacionados principalmente, à dificuldade de entendimento da prescrição, a erros de transcrição ou a problemas de estoque, detectados pelos farmacêuticos são:

$>\quad$ Duração do tratamento maior que a correta;

$>\quad$ Dose menor que a correta;

$>\quad$ Medicamento incorreto; transcrição incorreta;

$>\quad$ Duração do tratamento menor que a correta (inclui retirada precoce do tratamento);

$>\quad$ Dose maior que a correta;

> Medicamento incorreto: história prévia de alergia ou reação adversa; 


\author{
Forma farmacêutica errada; \\ Omissão na administração; \\ Medicamento necessário não prescrito (inclui profilaxia); \\ Omissão na dispensação.
}

Para Araújo et al., (2012), as medidas de redução de erros de medicação devem focar o sistema de medicação, a fim de evitar falhas humanas, e necessitam ser decididas em consonância com os demais profissionais, médicos e enfermeiros. Para garantir a segurança do paciente, todos devem trabalhar em conjunto e o farmacêutico deve assumir o papel de profissional clínico.

\title{
2. Metodologia
}

Trata-se de uma revisão integrativa da literatura baseada na pesquisa bibliográfica, que se configura como uma análise de pesquisas relevantes que dão suporte para a tomada de decisões e a melhoria da prática clínica, possibilitando a síntese do conhecimento de um determinado assunto, além de apontar lacunas do conhecimento que precisam ser preenchidas com a realização de novos estudos.

Segundo Galvão (2008) este método de pesquisa "permite a síntese de múltiplos estudos publicados e possibilita conclusões gerais a respeito de uma particular área de estudo".

Para a elaboração da revisão integrativa, algumas etapas foram seguidas: primeiramente, trabalhou-se a hipótese e a criação do objetivo do estudo, posteriormente foi selecionada a amostra através dos critérios de inclusão estabelecidos, tais como: ser artigo científico que não tenha como método de estudo revisão da literatura publicada na literatura nacional - no período 2014 a 2019 - que respondesse às questões da pesquisa no idioma português, disponível eletronicamente na íntegra. Foram excluídos os artigos que não atenderam ao objetivo proposto e aos critérios de seleção.

A definição da amostra do trabalho se deu através de pesquisa com fonte de busca eletrônica nos seguintes bancos de dados virtuais: Literatura Latino- Americana e do Caribe em Ciências da Saúde (LILACS) e Scientific Eletronic Library Online (SciELO). Para o levantamento dos artigos foram utilizados os seguintes descritores em saúde: atribuições; ambiente hospitalar; farmacêutico e segurança do paciente.

Após a busca dos artigos, obedecendo aos critérios de inclusão, as obras foram armazenadas em computador, para que em seguida fosse realizada uma pré-seleção de acordo com a leitura dos resumos. Nesta fase, foi verificada a relação entre os conteúdos, títulos, resumos e se atendem ao objetivo geral do estudo. Na fase de seleção, as obras foram lidas na íntegra com atenção especial para os resultados e conclusão dos estudos. Os estudos que não apresentaram qualquer relação com as atribuições do farmacêutico na promoção da segurança do paciente foram excluídos.

A análise dos resultados aconteceu por meio da categorização e apresentação em forma de quadros e tabelas do Microsoft Office Word 2010, esta etapa teve como finalidade sumarizar os resultados pertinentes a esta pesquisa e posteriormente discuti-los.

Foram extraídos dos artigos: autores, ano de publicação, cidade onde foi realizado o estudo, atribuições do farmacêutico, desenho do estudo, metodologia e resultados. Por último, foi realizada a análise dos textos e a realização de leitura interpretativa e redação.

As estratégias de busca permitiram a identificação de 571 artigos nas bases de dados selecionadas. Ao longo das análises dos títulos e resumos um total de 561 artigos foram excluídos por diversos motivos: assuntos não condizentes com o abordado, não apresentação de resumos ou artigos na íntegra e artigos em idiomas diferentes dos selecionados. Ao total foram 
selecionados dez artigos para análise do texto integral que participaram da revisão sobre o assunto. A figura 1 ilustra a estratégia de busca e o processo de seleção dos artigos.

Figura 1: Diagrama de fluxo da revisão integrativa da literatura.

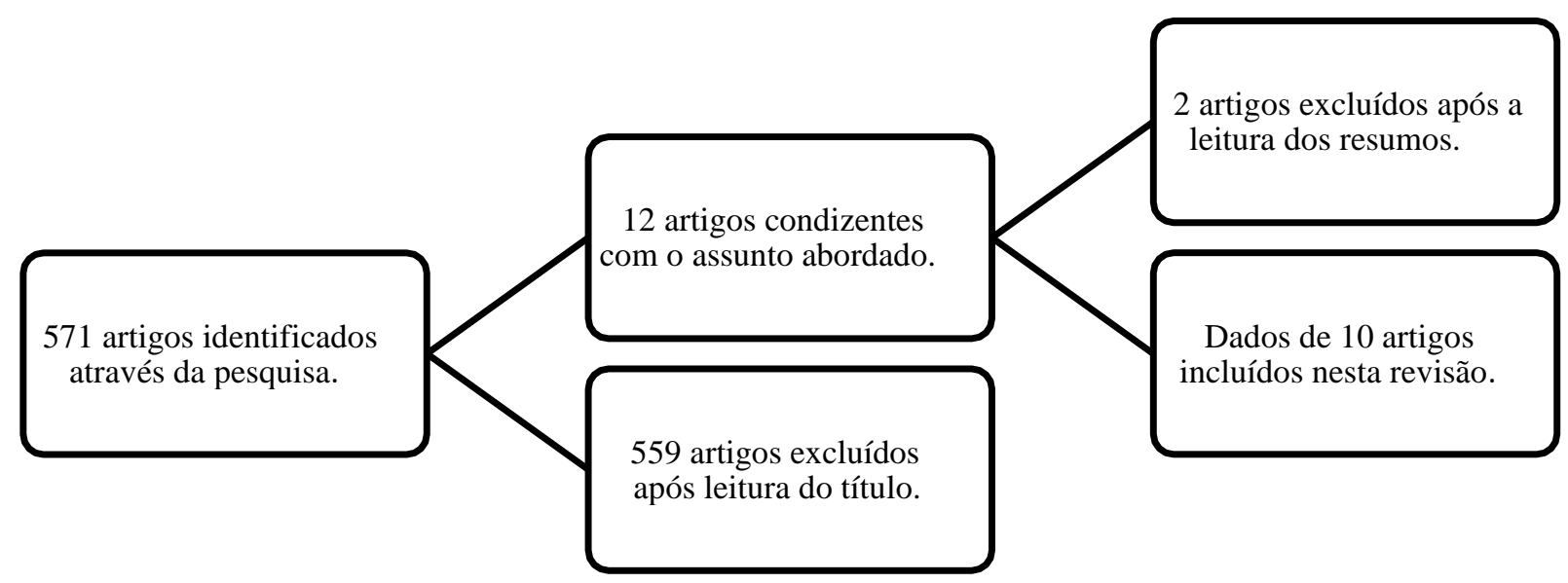

Fonte: Atribuições do farmacêutico no âmbito hospitalar para promoção da segurança do paciente: revisão integrativa da literatura, Redenção-Pará (2021).

\section{Resultados e Discussão}

O Quadro 1 apresenta detalhes dos 10 artigos selecionados. Todos os estudos foram publicados entre 2014 e 2019 e concluíram que a intervenção farmacêutica no uso de medicamentos melhora os desfechos dos pacientes internados. Todos os estudos foram conduzidos no Brasil. Os estudos analisaram a importância do farmacêutico clínico como membro da equipe multidisciplinar e suas principais contribuições para a segurança dos pacientes. 
Quadro 1: Descrição dos artigos selecionados.

\begin{tabular}{|c|c|c|}
\hline $\begin{array}{l}\text { Autor principal e ano de } \\
\text { publicação }\end{array}$ & Título do estudo & Objetivo geral \\
\hline Cardinal \& Fernandes (2014). & $\begin{array}{l}\text { Intervenção farmacêutica no processo de validação da } \\
\text { prescrição médica. }\end{array}$ & $\begin{array}{l}\text { Analisar as intervenções farmacêuticas realizadas durante a validação da prescrição médica e demonstrar o fluxo } \\
\text { do procedimento da validação da prescrição no hospital em estudo. }\end{array}$ \\
\hline Pezato \& Casaretti (2015) & $\begin{array}{l}\text { Farmacovigilância hospitalar: importância do treinamento } \\
\text { de profissionais na potencialização de suas ações. }\end{array}$ & $\begin{array}{l}\text { Identificar e caracterizar as notificações de reações, eventos adversos e desvios de qualidade de medicamentos em } \\
\text { hospital privado e treinar profissionais da saúde sobre farmacovigilância. }\end{array}$ \\
\hline Burigo \& Canabarro (2015) & $\begin{array}{l}\text { A cultura de segurança do paciente do serviço de farmácia } \\
\text { do Hospital Universitário/UFSC: um processo de formação. }\end{array}$ & $\begin{array}{l}\text { Compreender como se constitui o processo de desenvolvimento da cultura de segurança do paciente do Serviço de } \\
\text { Farmácia do Hospital Universitário da UFSC, a partir da visão dos seus gestores. }\end{array}$ \\
\hline Garske, et al. (2016) & $\begin{array}{l}\text { Avaliação das interações medicamentosas potenciais em } \\
\text { prescrições de pacientes em unidade de terapia intensiva. }\end{array}$ & Identificar e avaliar a existência de interações medicamentosas potenciais na farmacoterapia prescrita. \\
\hline Aguiar et al. (2017) & $\begin{array}{l}\text { Segurança do paciente e o valor da intervenção } \\
\text { farmacêutica em um hospital oncológico. }\end{array}$ & $\begin{array}{l}\text { Demonstrar o impacto econômico da avaliação farmacêutica na detecção e uma prevenção de erros em } \\
\text { prescrições de antineoplásicos. }\end{array}$ \\
\hline Farias e Assunção (2018) & $\begin{array}{l}\text { Avaliação da qualidade da prescrição em um hospital } \\
\text { terciário especializado em urgência e trauma. }\end{array}$ & $\begin{array}{l}\text { Avaliar a ocorrência dos erros de prescrição no setor de internação de um hospital de urgência/emergência da rede } \\
\text { pública brasileira, bem como identificar os tipos de erros e os medicamentos envolvidos. }\end{array}$ \\
\hline Souza et al. (2018) & $\begin{array}{c}\text { clínico no uso seguro e racional de medicamentos no } \\
\text { âmbito hospitalar. }\end{array}$ & Demonstrar a contribuição da farmácia clínica no uso seguro e racional de medicamentos. \\
\hline Carneiro et al. (2018) & $\begin{array}{l}\text { Segurança do paciente em unidade de terapia intensiva } \\
\text { neonatal: o impacto das recomendações farmacêuticas. }\end{array}$ & $\begin{array}{l}\text { Descrever as recomendações farmacêuticas realizadas durante a monitorização dos pacientes neonatos de uma } \\
\text { maternidade de Fortaleza, classificando-as e analisando- as quanto ao impacto, principais classes medicamentosas } \\
\text { e profissionais envolvidos, forma de contato e aceitação. }\end{array}$ \\
\hline Maioli \& Santos (2018) & $\begin{array}{c}\text { Intervenções farmacêuticas e sua importância na segurança } \\
\text { do paciente hospitalizado. }\end{array}$ & $\begin{array}{l}\text { Avaliar as intervenções farmacêuticos realizadas durante a análise dos prescrições médicas e a representatividade } \\
\text { do profissional farmacêutico na prevenção de erros relacionados a medicamentos. }\end{array}$ \\
\hline $\begin{array}{lllr}\begin{array}{l}\text { Deliberal, } \\
\text { (2019) }\end{array} & \text { Menezes \& Bueno } \\
\end{array}$ & $\begin{array}{c}\text { Segurança do paciente na implantação de dispensários } \\
\text { eletrônicos: análise da função "override". }\end{array}$ & $\begin{array}{l}\text { Analisar os medicamentos dispensados em dois dispensários eletrônicos, sendo um com função "override" } \\
\text { ativada e outro sem a função ativada. }\end{array}$ \\
\hline
\end{tabular}

Fonte: Atribuições do farmacêutico no âmbito hospitalar para promoção da segurança do paciente: Revisão integrativa da literatura, Redenção - Pará, 2021. 
O primeiro estudo selecionado apresenta um estudo unicêntrico, observacional, descritivo e transversal em um hospital privado. Realizou-se a quantificação das intervenções farmacêuticas realizadas durante o processo de validação da prescrição médica medicamentosa. Os autores apontam que as intervenções farmacêuticas realizadas anteriormente a dispensação de medicamentos são efetivas na prevenção de erros de medicação. A aplicação de sistema de validação é visto como garantia de maior segurança do paciente e qualidade do usuário internado (Cardinal \& Fernandes, 2014).

Outro estudo selecionado objetivou identificar e caracterizar as notificações de reações, eventos adversos e desvios de qualidade de medicamentos em hospital privado e treinar profissionais da saúde sobre farmacovigilância. Os autores apresentam os resultados de um estudo seccional e quantitativo conduzindo em um hospital privado no município de Sorocaba, no Estado de São Paulo. Neste estudo verificou-se que no curto treinamento aplicado sobre farmacovigilância em um hospital privado, resultou num aumento expressivo do número de notificações e apontou ainda fragilidade nas unidades de internação em identificar reações

adversas e medicamentos.

De acordo com Pezato e Cesaretti (2015) o treinamento dos profissionais de saúde sobre o tema de farmacovigilância aumentou o número de notificações de reações adversas e eventos adversos a medicamentos nas unidades de internação, aumentando com isso, a segurança dos usuários em processo de internação hospitalar.

Em outra publicação, incluída no presente estudo, os autores investigaram a constituição e o processo de desenvolvimento da cultura de segurança do paciente do serviço de farmácia do Hospital Universitário da Universidade Federal de Santa Catarina, a partir da visão dos seus gestores. A pesquisa caracterizou-se por um estudo de caso, de natureza qualitativa. Os autores identificaram, através dos resultados obtidos, que o serviço de farmácia do referido hospital vem vivenciando na prática ações, ainda que incipientes no contexto institucional, para o desenvolvimento do processo da cultura de segurança do paciente. Quanto aos desafios e possibilidades de desenvolvimento do processo de cultura de segurança do paciente, os gestores pontuaram a deficiência da oferta de cursos de capacitação sobre segurança do paciente (Burigo \& Canabarro, 2015).

Considerando as evidências disponíveis, foi possível verificar em quatro estudos de natureza retrospectiva, observacional e descritiva a importância da atuação do farmacêutico hospitalar no tocante à interações medicamentosas; intervenções farmacêuticas específicas e sua importância na segurança do paciente; avaliação, pelo farmacêutico, da qualidade das prescrições medicamentosas e a sobre a importância o farmacêutico no uso seguro e racional de medicamentos no âmbito hospitalar. Alinhado com Costa, Togeiro e Ribeiro (2013) os farmacêuticos são fundamentais para garantir o uso racional e seguro dos medicamentos, bem como alertar quanto aos erros de medicação e como preveni-los. Eles podem trazer contribuições significativas à equipe que atua em farmácia hospitalar, muito além do simples papel de dispensador de medicamentos. Ainda para os autores, a atenção farmacêutica para existir dentro do hospital tem que haver a conjugação com o trabalho de outros profissionais. Ou seja, o paciente recebe cuidados de grandes números de pequenos cuidados parciais que se complementam na interação de outros cuidadores que trabalham no hospital.

Aguiar et al., (2017) reafirmam a importante contribuição do farmacêutico nas ações de promoção, proteção e recuperação da saúde, especialmente quanto à análise de prescrição. A segurança do paciente, ainda segundo os autores, é um alvo dinâmico, e a abordagem farmacêutica para alcança-la devem continuar evoluindo para melhorar cada vez mais a assistência farmacêutica prestada.

O farmacêutico clínico traz benefícios através da identificação de potenciais eventos adversos relacionados a terapia medicamentosa e consequentemente, contribui para a segurança do paciente no desfecho positivo do paciente. Apesar da importância já bem estabelecida do farmacêutico clínico, os autores, relatam que experiências vêm discretamente sendo 
relatadas e a importância desses serviços para a criança, adolescente e adulto tem levado a resultados interessantes (Becker \& Bueno, 2018).

Em outra publicação selecionada, os autores analisaram a segurança do paciente na implantação de dispensários eletrônicos. Trata-se de um estudo transversal. Os autores identificaram passíveis fragilidades existentes no sistema dos dispensários eletrônicos e sinalizam a necessidade de monitoramento contínuo do uso dessa tecnologia. Para Deliberal, Menezes e Bueno (2018) o uso de dispensários eletrônicos contribui com a precisão e eficiência dos processos logísticos, mas não impedem erros relacionados a medicamentos aconteçam. $\mathrm{O}$ objetivo principal dos dispensários eletrônicos é permitir o acesso a medicamentos em situações de urgência e emergência, locais sem farmácias 24 hora e unidades de emergência. $O$ uso inadequado é baseado em padrões de prática, percepções de que a farmácia não pode processar a prescrição médica tão rapidamente quanto necessário e com prescrições médicas verbais.

De acordo com um dos estudos revisados, as intervenções farmacêuticas realizadas antes da dispensação de medicamentos são efetivas na prevenção de erros de medicação. A implantação de sistemas de validação da prescrição médica, segundo os autores, é garantia de maior segurança e qualidade do tratamento ao paciente internado. Contudo, no Brasil, estudos sobre intervenção farmacêutica em hospitais são escassos, principalmente em instituições privadas, portanto o presente estudo contribui para o avanço do conhecimento da atuação do farmacêutico clínico no país atuando junto à equipe multidisciplinar para o aprimoramento da segurança dos pacientes.

\section{Considerações Finais}

O desenvolvimento da assistência farmacêutica é um grande avanço. Está relacionada à tecnologia de gestão de medicamento, para garantia de acesso; e, também relacionada à tecnologia do uso do medicamento, que prevê utilização correta do medicamento, portanto, a atenção farmacêutica pode ser considerada atividade específica do farmacêutico, no âmbito hospitalar.

Considerando técnicas e instrumentos propostos nos estudos incluídos, se constatou certa homogeneidade, uma vez que todos os estudos abordam a importância do profissional farmacêutico presente na rotina hospitalar e sendo um dos principais responsáveis pela segurança dos pacientes internados e, principalmente àqueles que estão recebendo terapia medicamentosa.

Constata-se que na atualidade, é necessário um farmacêutico, para o acompanhamento dos usuários hospitalizados, por isso o farmacêutico precisa pensar na sua relação profissional/paciente. Ações simples de serem implantadas, como intervenção farmacêutica a partir da análise de prescrições, podem identificar problemas relacionados a medicamentos, prevenir eventos adversos, reduzir perdas financeiras e agregar imensurável valor na segurança do paciente.

Todos os estudos demonstram que a presença do farmacêutico exerce uma interferência benéfica às prescrições médicas, melhorando assim, a qualidade dos serviços prestados ao paciente, diminuindo o óbito, diminuindo o número e o tempo de hospitalizações e reduz os custos hospitalares.

\section{Contribuições dos autores}

MEDS concebeu a estratégia de pesquisa em conjunto com YJM e AEMO. MEDS fez as buscas bibliográficas nas bases de dados, a seleção dos artigos e a extração de dados sob a supervisão de YJM e AEMO. Os autores leram e aprovaram a versão final do manuscrito. O conteúdo da revisão é de exclusiva responsabilidade individuais dos autores. 


\section{Declaração de conflito de interesses}

Os autores declaram que a pesquisa foi conduzida na ausência de quaisquer relações comerciais ou financeiras que possam ser interpretados como um potencial conflito de interesse.

\section{Referencias}

Aguiar, K. D. S., Santos, J. M. D., Cambrussi, M. C., Picolotto, S., \& Carneiro, M. B. (2018). Segurança do paciente e o valor da intervenção farmacêutica em um hospital oncológico. Einstein, 16. https://www.scielo.br/j/eins/a/ZpPshMSx9tcJYTT3yzqMXSP/?format=html\&lang=pt.

Alcântara, S. T., \& Cassiolato, S. (2010). Segurança do paciente: a atuação da farmácia na prevenção de erros de medicação em unidade de emergência de um hospital universitário [dissertação]. Ribeirão Preto: Universidade de São Paulo, Faculdade de Medicina de Ribeirão Preto. http://bvsms.saude.gov.br/bvs/premio_medica/2010/mencoes/trabalho_completo_si reli_teresinha_alcantara.pdf.

Galvão, A. A., de Oliveira, A. M., de Carvalho, F. B., \& Araújo, R. P. C. (2012). Identificação e distribuição dos erros de dispensação em uma farmácia hospitalar: um estudo comparativo no município de Salvador Bahia. Revista de Ciências Médicas e Biológicas, 11(2), 201-206.

National Academies of Sciences, Engineering, and Medicine. (2015). Improving diagnosis in health care. National Academies Press.

Bohrer, C. D., Marques, L. G. S., Vasconcelos, R. O., Oliveira, J. L. C. D., Nicola, A. L., \& Kawamoto, A. M. (2016). Comunicação e cultura de segurança do paciente no ambiente hospitalar: visão da equipe multiprofissional. Rev. enferm. UFSM, 50-60. https://pesquisa.bvsalud.org/portal/resource/pt/bde-31909.

de Farmácia, C. F. (2013). Resolução CFF n 586, de 29 de Agosto de 2013. Regula a prescrição Farmacêutica e dá outras providências. Diário Oficial da União, 29. http://www.cff.org.br/userfiles/file/resolucoes/357.pdf.

de Farmácia, C. F. (2013). Resolução CFF no 586, de 29 de Agosto de 2013. Regula a prescrição Farmacêutica e dá outras providências. Diário Oficial da União, 29. http://www.cff.org.br/userfiles/file/resolucoes/585.pdf.

Sousa, P., \& Mendes, W. (2019). Segurança do paciente: criando organizações de saúde seguras. Editora Fiocruz. http://books.scielo.org/id/bskw2.

Brasil. (2013). Ministério da Saúde. Protocolo de segurança na prescrição, uso e administração de medicamentos. http://www.saude.pr.gov.br/arquivos/File/OSEGURANCA_DO_PACIENTE/PROTOC OLOSEGURANAMEDICAMENTOSA.pdf>.

Bueno, A. A. B., \& Fassarella, C. S. (2012). Segurança do Paciente: uma reflexão sobre sua trajetória histórica. Revista Rede de cuidados em Saúde, 6(1). http://publicacoes.unigranrio.edu.br/index.php/rcs/article/view/1573.

Burigo, C. C. D., \& Isabel Machado, C. A. N. A. B. A. R. R. O. (2015). A Cultura De Segurança Do Paciente Do Serviço De Farmácia Do Hospital Universitário/Ufsc: Um Processo Em Formação. https://repositorio.ufsc.br/handle/123456789/135794.

Cardinal, L. D. S. M., \& Fernandes, C. S. (2014). Intervenção farmacêutica no processo da validação da prescrição médica. Revista Brasileira de Farmácia Hospitalar e Serviços de Saúde, 5(2). https://rbfhss.org.br/sbrafh/index.

Carneiro, A. I. C., \& Silva, A. H. (2018). Segurança do paciente em unidade de terapia intensiva neonatal: o impacto das recomendações farmacêuticas. Conexão Fametro. https://www.doity.com.br/media/doity/submissoes/artigo25f9e0711ed1f5389c75301 87afde129c955b4e2-arquivo.pdf.

Carvalho, M. D., \& Vieira, A. A. (2002). Erro médico em pacientes hospitalizados. Jornal de Pediatria, 78, 261-268. https://www.scielo.br/j/jped/a/RG4mGKjYCrCvp84bD79MX8s/abstract/?stop=previous\&lang=pt\&format=html.

Cassiani, S. H. D. B. (2000). Erros na medicação: estratégias de prevenção. Revista Brasileira de Enfermagem, 53(3), 424-430. https://www.scielo.br/j/reben/a/B7X5wcr5t3z7TRttSpPms3r/?lang=pt.

Nunes, P. H. C., Pereira, B. M. G., Nominato, J. C. S., Albuquerque, E. M. D., Silva, L. D. F. N. D., Castro, I. R. S. D., \& Castilho, S. R. D. (2008). Intervenção farmacêutica e prevenção de eventos adversos. Revista Brasileira de Ciências Farmacêuticas, 44, 691-699. https://www.scielo.br/j/rbcf/a/c8VRrXsB3brGrfvPhrpdNFk/abstract/?lang=pt.

Deliberal, A. P., Menezes, C. P., \& Bueno, D. (2019). Segurança do paciente na implantaçãode dispensários eletrônicos: análise dafunção" override". Revista da OFIL-Ibero Latin America Journal of Health System Pharmacy. Madrid. 29(1), 43-47. https://www.lume.ufrgs.br/handle/10183/194822.

Dantas, S. C. C. (2011). Farmácia e controle das infecções hospitalares. Pharmacia brasileira, 1(80), 1-20. http://www.saocamilo-sp.br/novo/eventosnoticias/simposio/14/SCF001_14.pdf.

Farias, P. D. O., \& Assunçao, A. L. F. D. Avaliação da qualidade da prescrição em um hospital terciário especializado em urgência e trauma. http://www.rmmg.org/artigo/detalhes/2433.

Ferracini, F. T., \& BORGES FILHO, W. M. (2010). Prática farmacêutica no ambiente hospitalar: do planejamento à realização. Atheneu.

Garske, C. C. D., Brixner, B., Freitas, A. P., \& Schneider, A. P. H. (2016). Avaliação das interações medicamentosas potenciais em prescrições de pacientes em unidade de terapia intensiva. Saúde e Pesquisa, 9(3), 483-490. https://periodicos.unicesumar.edu.br/index.php/saudpesq/article/view/5399.

Maioli, N. A., \& Santos, H. C. B. (2018, August). Intervenções farmacêuticas e sua importância na segurança do paciente hospitalizado. In Colloquium Vitae. 10(2), 35-40. https://revistas.unoeste.br/index.php/cv/article/view/1531. 
Research, Society and Development, v. 10, n. 13, e544101320566, 2021

(CC BY 4.0) | ISSN 2525-3409 | DOI: http://dx.doi.org/10.33448/rsd-v10i13.20566

Nascimento, J. C., \& Draganov, P. B. (2015). História da qualidade em segurança do paciente. História da Enfermagem revista eletrônica, 6(2), 299-309. http://here.abennacional.org.br/here/seguranca_do_paciente.pdf.

Pezato, T. P. J., \& Cesaretti, M. L. R. (2015). Farmacovigilância hospitalar: importância do treinamento de profissionais na potencialização de suas ações. Revista da Faculdade de Ciências Médicas de Sorocaba, 17(3), 135-139. https://revistas.pucsp.br/RFCMS/article/view/23518.

Ribeiro, V. F., Sapucaia, K. C. G., Aragão, L. A. O., Bispo, I. C. D. S., Oliveira, V. F., \& Alves, B. L. (2015). Realização de intervenções farmacêuticas por meio de uma experiência em farmácia clínica. Revista Brasileira de Farmácia Hospitalar e Serviços de Saúde,6(4). https://www.rbfhss.org.br/sbrafh/article/view/238.

de Souza, L. B., de Souza, D. M., de Souza, S. M., da Silva, D. R., \& Aguilar, N. C. (2018). Importância do farmacêutico clínico no uso seguro e racional de medicamentos no âmbito hospitalar. Pensar Acadêmico, 16(1), 109-124. http://pensaracademico.facig.edu.br/index.php/pensaracademico/article/view/360.

Wachter, R. M. (2013). Compreendendo a segurança do paciente. AMGH Editora. https://books.google.com.br/books?hl=ptBR\&1r=\&id=LWQ3AgAAQBAJ\&oi=fnd\&pg=PR3\&dq=Compreendendo+a+seguran\%C3\%A7a+do+paciente\&ots=sCwqbfeXAi\&sig=eEf63_V_xEoWjIgfyvcaNuPLdw\#v=onepage $\& q=$ Compreendendo $\% 20 \mathrm{a} \% 20$ seguran $\% \mathrm{C} 3 \% \mathrm{~A} 7 \mathrm{a} \% 20 \mathrm{do} \% 20$ paciente $\& \mathrm{f}=$ false 